American Journal of Environmental Sciences 7 (6): 510-514, 2011

ISSN 1553-345X

(C) 2011 Science Publications

\title{
Analysis of Eco friendly Refrigerants Usage in Air-Conditioner
}

\author{
${ }^{1}$ Chinnaraj, C., ${ }^{1}$ R. Vijayan and ${ }^{2}$ P. Govindarajan \\ ${ }^{1}$ Department of Mechanical Engineering, \\ Government College of Engineering, Salem, India \\ ${ }^{2}$ Department of Mechanical Engineering, \\ Sona College of Technology, Salem, India
}

\begin{abstract}
Problem statement: There are two types of global warming contributions through refrigeration and air conditioning systems. The first one is the Direct Global Warming Potential (DGWP) due to the emission of refrigerants and their interaction with heat radiation. The second one is the Indirect Global Warming Potential (IDGWP) due to the emission of Carbon Dioxide $\left(\mathrm{CO}_{2}\right)$ by consuming the energy that is generated through the combustion of fossil fuels. Most of refrigerants used in vapor Compression system were Chlorofluorocarbon (CFCs) and Hydro Chlorofluorocarbon (HCFCs) which contains chlorine and if any leakage in the system, these gases will go up and reach stratosphere. The chlorine atoms in the gases will act as a catalyst to destroy ozone layer and cause ozone depletion which causes health hazards, global warming, melting of polar ice caps and drought. Hence, it is necessary to minimize the Global warming and Ozone depletion. The refrigerant R22 widely used in the airconditioners is a major Contributor of Chlorofluorocarbons (CFCs) which cause irreparable loss to the ozone layer and has to be replaced. Approach: To conserve the energy and minimize the global warming, the systems should be designed as more energy efficient and also to minimize Ozone depletion, the eco friendly refrigerants are to be selected and tested as alternative refrigerants to R22. Hence, a window air conditioner of $3.5 \mathrm{~kW}$ capacity fitted with Electronic Expansion Valve (EEV)) instead of capillary tube as an expansion device, was tested for its performance with the selected eco friendly refrigerants R407C and R290 as an alternative to R22 under fixed indoor and outdoor chamber temperatures in the experimental set up and varying the EEV opening. Results: It has been observed from the experimental studies that when the smaller capacity R22 window air conditioner with EEV is retrofitted with R407C and R290, compared to the performance given by R22, the Coefficient Of Performance (COP) given by R407C and R290 is improved in the range of 4.8-7.1 and 8.0-12.3\% respectively and the Energy Efficiency Ratio (EER) is increased by $4.2-6.9 \%$ for R407C and $6.4-10.8 \%$ for R290. Conclusion: The performance of Electronic Expansion Valve (EEV) with eco friendly refrigerant R290 in the given air conditioner shows a positive effect in terms of COP and energy efficiency and enables the industry to favorably displace the R22 and other types of expansion devices.
\end{abstract}

Key words: Coefficient Of Performance (COP), Indirect Global Warming Potential (IDGWP), Direct Global Warming Potential (DGWP), Electronic Expansion Valve (EEV), ChloroFluorocarbon (HCFCs), Energy Efficiency Ratio (EER)

\section{INTRODUCTION}

Worldwide attempts are being made to phase out the production and consumption of Hydro ChloroFluorocarbon (HCFCs) and Chlorofluorocarbon CFCs), as these chemicals are responsible for depletion of stratospheric ozone layer. Refrigeration, Airconditioning and heat pumps sectors are one of the principal users of these chemicals. As per Montreal Protocol (1987), refrigerant R22, the generally accepted and most suitable refrigerant for air conditioners must be phased out by 2030 by developed countries and by 2040 by developing countries because of its Ozone Depleting Potential. The phasing out of ozone depleting refrigerants has led to the quest for eco-friendly alternative refrigerants and substitutes for R22 have been developed.

Since the demand for air conditioners by the people are increasing throughout the world day by day, considering the indirect Global Warming and Ozone Depletion, it is an urgent need to improve the energy efficiency of vapor compression system as it is

Corresponding Author: Chinnaraj, C., Department of Mechanical Engineering, Government College of Engineering, Salem, India 
most widely used in majority of modern cooling equipments.

With the above objectives in mind a detailed literature survey has been carried out and the possibility of adopting Electronic Expansion Valve (EEV) instead of capillary tube as an expansion device in lower capacity window air conditioners with eco friendly refrigerant $\mathrm{R} 407 \mathrm{C}$ and R 290 as alternative refrigerant to R22 to improve the energy efficiency of the system is tested.

Alternate refrigerants: Calm (2008) compiled the historical developments of pure refrigerants from early use as first generation (from the year 1830 to1930) to the present as fourth generation and also addressed future options based on low Global Warming Potential (GWP) and zero or low Ozone Depletion Potential (ODP). Air-conditioning and Refrigeration Institute ARI, 1997, Arlington, VA, USA, in the year 1997 suggested that the refrigerants R407C and R290 are the potential replacements for HCFC-22. Among these two, R290 is a byproduct of natural gas that can serve as a replacement of CFCs and HCFCs. Its liquid pressure is higher than that of $\mathrm{HCFC} 22$ and hence the performance increases significantly. The better vapor compression system performance of R290 is attributed to high evaporation temperature and compressor work. It is also noted that the performance of R290 is higher than R22 under all working conditions. Mohanraj et al. (2011) suggested a list of refrigerants as alternative to R22 and their properties.

The problem of R22 substitution in terms of global warming effect in vapour compression refrigeration effect was examined and reported that at higher condensation (over $50^{\circ} \mathrm{C}$ ) and evaporation temperature $\left(2-10^{\circ} \mathrm{C}\right)$, the Total Equivalent Warming Impact (TEWI) of R407C is even slightly lower than of R22 and its substitution for R22 is convenient from the point of view of the green house effect (Aprea and Greco, 2003).

Devotta et al. (2005) tested R290 as alternative to $\mathrm{R} 22$ in a $5.17 \mathrm{~kW}$ (1.5TR) window air conditioner and reported that cooling capacity was $6.6-9.7 \%$ lower and energy consumption was $12.4-13.5 \%$ lower than that of R22. But COP was 2.8-7.9\% higher than that of R22. United Nation's Environmental Program (UNEP)'s Technology and Economic Assessment Panel (TEAP) Unep Teap Progress Report, 2010 (decision XIX /8) stated that HC-290 has successfully been commercialized as an $\mathrm{HCFC}-22$ replacement in low charge, room and portable air -conditioner applications of less than $4 \mathrm{~kW}$.

Advantages of EEV: Among the different expansion devices used in the vapor compression refrigeration cycle, EEV shows the better overall performance due to the regulation and modulation of the refrigerant feed through the valve is accomplished through a micro processor based control (electronic temperature sensors) and an electric motor operating the valve orifice size. Study by (Aprea and Mastrullo, 2002) showed that for the refrigerants R22 and R407C used in a vapour compression plant, an overall better performance of EEV compared with the TEV under transient conditions, while under steady state conditions both the valves are equal in performance.

For the refrigerants R22 and R417A, adopting EEVs in air conditioners enables an appreciable energy saving with respect to the same installations equipped with traditional Thermostatic Expansion Valves (TEVs) was proved and this is due to the fact that EEVs allow a lower condensation pressure in systems equipped with air cooled condensers, which is adjusted to variations in outside air temperature (Lazzarin and Noro, 2008). Shanwei et al. (2005) have developed a correlation on the basis of the experimental data of EEV to predict the mass flow rate for $\mathrm{R} 22$ and its alternatives, R407C and R410A.

\section{MATERIALS AND METHODS}

An experimental setup was designed to measure the performance of the window air conditioner under variable operating conditions. The nominal cooling capacity of the window air conditioner is $3.5 \mathrm{~kW}$ using R22, R407C and R290 as working fluids. The window air conditioner consists of Kirloskar made $3.5 \mathrm{~kW}$ capacity hermetic type compressor, two heat exchangers (condenser and evaporator), Danfoss made EEV of 2.3 m.m orifice diameter and 0-2625 steps and environmental chambers to simulate the indoor and outdoor conditions and the necessary instrumentation. It is modified to accommodate various sensors and Coriolis type mass flow meter. All sensors are connected to a computerized data acquisition system (AGILENT 34970A). Figure 1 shows the photograph of the test facility. Test conditions were maintained according to Bureau of Indian Standards (BIS) (1992) 1391 standards Bureau of Indian Standards, 1992.

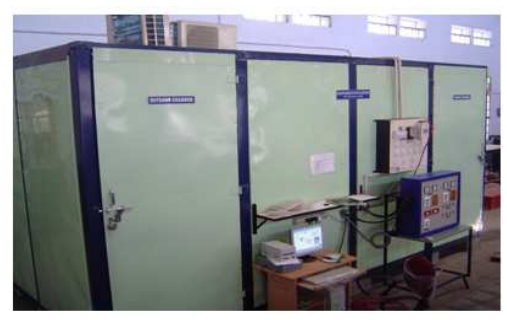

Fig. 1: Photograph of the test facility 
For all the tests, the infiltration heat load is added to the readings obtained from the energy meter to compute the actual capacity of the system under a particular test condition.

By keeping the indoor chamber temperature and outdoor chamber temperature of the experimental test facility as 25 and $40^{\circ} \mathrm{C}$ respectively and varying the EEV opening as $15 \%$ (416 steps), $20 \%$ (546 steps) and $25 \%$ (676 steps), the experiment was carried out for the refrigerant R22. The experiment was repeated again by retrofitting the compressor with $\mathrm{R} 407 \mathrm{C}$ and then with R290 by keeping the test conditions as before. Corresponding readings were noted. From the readings, the performance parameters such as Coefficient Of Performance (COP) and Energy Efficiency Ratio (EER) were calculated and analyzed.

\section{RESULTS}

Mass flow rate: Figure 2 shows the variation of mass flow rate of refrigerants with EEV opening for the given indoor chamber and outdoor chamber temperatures. The mass flow rate of R22, R407C and R290 are increasing with the increase in EEV opening from $15 \%$ (416 steps) to $20 \%$ (546 steps) opening and then decreasing for the EEV opening of 25\% (676 steps).

For the entire test, mass flow rate of all the refrigerants are maximum at 20\% (546 steps) EEV opening and compared to mass flow rate of R22, the mass flow rate of $\mathrm{R} 407 \mathrm{C}$ is higher in the range of 20.2$63.7 \%$. The mass flow rate of R290 is lower than that of $\mathrm{R} 22$ in the range of $50.8-60.7 \%$ and lower than that of $\mathrm{R} 407 \mathrm{C}$ in the range of $61.25-70 \%$.

Compressor power input: The variation of compressor power input for the refrigeration system with EEV opening for the given indoor chamber and outdoor chamber temperatures are given in the Fig. 3. The compressor power input of the system for R22, $\mathrm{R} 407 \mathrm{C}$ and R290 is increasing with increase in EEV opening from $15 \%$ (416 steps) to $20 \%$ (546 steps) opening and then decreasing for the EEV opening of $25 \%$ (676 steps).

For the entire test, compressor power input of the system for all the refrigerants are maximum at $20 \%$ (546 steps) EEV opening and compressor power input of the system for R290 is lower than that of R22 in the range of 10.84-13.2\% and lower than that of R407C in the range of 8.8-10.6\%. The compressor power input of the system for $\mathrm{R} 407 \mathrm{C}$ is lower than that of R22 in the range of $2.1-13.2 \%$.

Coefficient of performance: Figure 4 represents the variation of COP of the system for the refrigerants with
EEV opening for the given indoor chamber and outdoor chamber temperatures. The COP of the system for R22, $\mathrm{R} 407 \mathrm{C}$ and R290 is increasing with increase in EEV opening from $15 \%$ (416 steps) to $20 \%$ (546 steps) opening and then decreasing for the EEV opening of $25 \%$ (676 steps).

For the entire test, COP of the system for all the refrigerants are maximum at 20\% (546 steps) EEV opening and the COP of the system for R290 is higher than that of R22 in the range of $8.0-12.3 \%$ and higher than that of $\mathrm{R} 407 \mathrm{C}$ in the range of $2.0-6.6 \%$. The COP of the system for $\mathrm{R} 407 \mathrm{C}$ is higher than that of R22 in the range of $4.8-7.1 \%$. COP is found to be the maximum with R290.

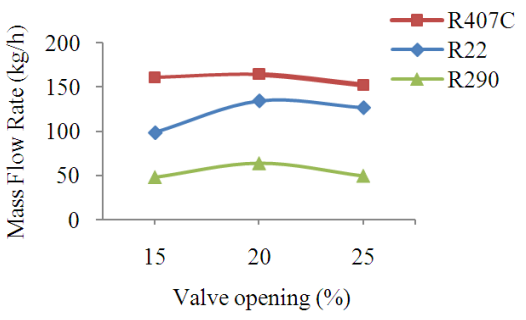

Fig. 2: Effect of EEV opening on the mass flow rate at outdoor chamber temperature of $40^{\circ} \mathrm{C}$

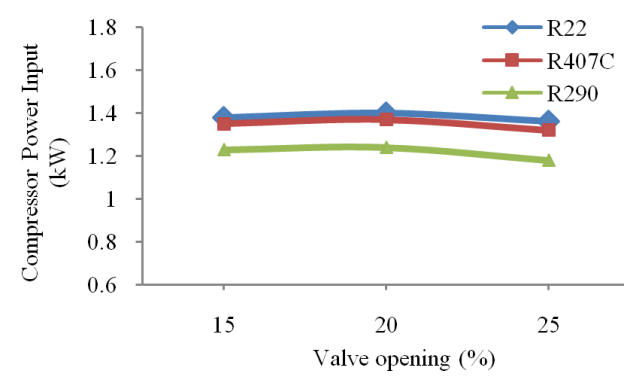

Fig. 3: Effect of EEV opening on the compressor power input of the system at outdoor chamber temperature of $40^{\circ} \mathrm{C}$

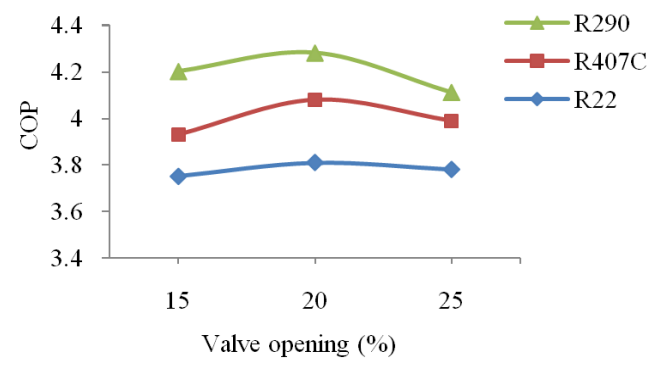

Fig. 4: Effect of EEV opening on the COP at outdoor chamber temperature of $40^{\circ} \mathrm{C}$ 
Am. J. Environ. Sci., 7 (6): 510-514, 2011

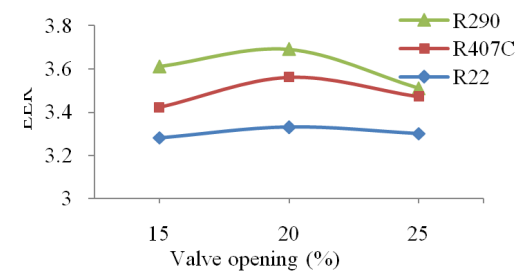

Fig. 5: Effect of EEV opening on the EER at outdoor chamber temperature of $40^{\circ} \mathrm{C}$

Energy efficiency ratio: The variation of EER of the system for the refrigerants with EEV opening for the given indoor chamber and outdoor chamber temperatures are given in the Fig. 5. The EER of the system for R22, R407C and R290 is increasing with increase in EEV opening from 15\% (416 steps) to 20\% (546 steps) opening and then decreasing for the EEV opening of $25 \%$ ( 676 steps).

For the entire test, EER of the system for all the refrigerants are maximum at 20\% (546 steps) EEV opening and the EER of the system for R290 is higher than that of R22 in the range of 6.4-10.8\% and higher than that of $\mathrm{R} 407 \mathrm{C}$ in the range of $0.8-5.6 \%$.The EER of the system for R407C is higher than that of R22 in the range of $4.2-6.9 \%$. EER of the system is found to be the maximum with R290.

\section{DISCUSSION}

From the above test, values of all the performance parameters are increasing with increase in valve opening upto $20 \%$ (546 steps) and then decreasing due to increase in mass flow rate of refrigerant upto $20 \%$ EEV opening and then decreasing.Even though the steps or valve opening is increased after 20\% (546 steps), the valve operating mechanism is not allowing higher mass flow rate of refrigerant due to limited capacity of airconditioner. For the entire test, the reduction in mass flow rate for R290 can be attributed to its lower liquid density compared to R22and R407C. Compressor power input for the R290 system is found to be the minimum due to its lower mass flow rate and lower condenser operating pressure.

Even though the cooling capacity and mass flow rate are found to be less for R290, a considerable reduction in compressor power input effects in improved COP and EER values for the entire range of EEV opening.

\section{CONCLUSION}

From the experiments carried out to compare the performances of R22, R407C and R290 using the EEV in a small window air conditioner of $3.5 \mathrm{~kW}$ capacity the following conclusion is drawn:

- Mass flow rate and cooling capacity of R290 system with EEV are lower than that of R22and R407C system with EEV due to its lower liquid density and lower latent heat of vaporization

- Power consumption by R290 system is always lower than that of R22 and R407C system due to its lower mass flow rate and lower pressure ratio

- R290 system has higher COP than R22 system and $\mathrm{R} 407 \mathrm{C}$ system in the range of $8.0-2.3 \%$ and $2.0-$ $6.6 \%$ respectively. Also it is having higher EER than R22 system and R407C system in the range of $6.4-10.8 \%$ and $0.8-5.6 \%$ respectively due to its lower power input

- The system is giving its best performance at EEV opening of $20 \%$ (546 steps)

- Owing to negligible Ozone Depletion Potential and lower Global Warming Potential, R290 can serve as functional replacement for R22 in conventional Refrigeration and Air-conditioning systems by following the International safety standards (IEC 60335-2-24,-2-34,-2-89,-2-40)

\section{REFERENCES}

Aprea, C. and R. Mastrullo, 2002. Experimental evaluation of electronic and thermostatic expansion valves performances using R22 and R407C. Applied Thermal Eng., 22: 205-218. DOI: 10.1016/S1359-4311(01)00071-0

Aprea, C. and A .Greco, 2003. Performance evaluation of R22 and R407C in a vapour compression plant with reciprocating compressor. Applied Thermal Eng., 23: 215-227. DOI: 10.1016/S13594311(02)00160-6

Calm, J.M., 2008. The next generation of refrigerantsHistorical review, considerations and outlook. Int. J. Refrigeration, 31: 1123-1133. DOI: 10.1016/j.ijrefrig.2008.01.013

Devotta, S., A.S. Padalkar and N.K. Sane, 2005. Performance assessment of HC-290 as a drop-in substitute to HCFC-22 in a window air conditioner. Int. J. Refrigeration, 28: 594-604. DOI: 10.1016/j.ijrefrig.2004.09.013

Lazzarin, R. and M. Noro, 2008. Experimental comparison of electronic and thermostatic expansion valves performances in an air conditioning plant. Int. J. Refrigeration, 31: 113118. DOI: 10.1016/j.ijrefrig.2007.09.004 
Mohanraj, M., C. Muraleedharan and S. Jayaraj, 2011. A review on recent developments in new refrigerant mixtures for vapour compression-based refrigeration, air-conditioning and heat pump units. Int. J. Energy Res., 35: 647-669. $\quad$ DOI: 10.1002/er.1736
Shanwei, M., Z. Chuan, C. Jiangping and C. Zhiujiu, 2005. Experimental research on refrigerant mass flow coefficient of electronic expansion valve. Applied Thermal Eng., 25: 2351-2366. DOI: 10.1016/j.applthermaleng.2004.12.005 